\title{
Acute Plasmodium knowlesi Infection in Olive Baboons (Papio anubis) Is Accompanied by High-Level of Gamma Interferon
}

\author{
Teresia Nyawira (Corresponding author) \\ National Council for Science and Technology \\ PO box 30623 - 00100, Nairobi, Kenya
}

Tel: 254-722-560-809 E-mail: teresianyawira@yahoo.com

Michael Gicheru

Kenyatta University, School of Pure and Applied Sciences

PO box 43844 - 00100, Nairobi, Kenya

Tel: 254-722-609-765Ｅ-mail:muitaku@gmail.com

\section{Esther Kagasi}

Institute of Primate research, Department of Tropical and Infectious Diseases

PO box 24481, Karen, Nairobi, Kenya

Tel: 254-733-627-854Ｅ-mail: estherkagasi@yahoo.com

Zipporah Ng'ang'a

Jomo Kenyatta University of Agric. and Tech

Institute of Tropical medicine and Infectious Diseases

PO box 62000 - 00200, Nairobi, Kenya

Tel: 254-722-794-883_E-mail: zipnganga2002@yahoo.com

Hastings Ozwara

Institute of Primate Research, Department of Tropical and Infectious Diseases

PO box 24481, Karen, Nairobi, Kenya

Tel: 254-721-483-326_E-mail: ozwara@primateresearch.org

Received: February 1, $2011 \quad$ Accepted: March 9, $2011 \quad$ Published: April 1, 2012

doi:10.5539/ijb.v4n2p92

URL: http://dx.doi.org/10.5539/ijb.v4n2p92

This research received financial support under the Multilateral Initiative on Malaria (MIM) Project ID A50075 through the UNICEF/UNDP/World Bank/WHO Special Programme for Research and Training in Tropical Diseases (TDR).

\begin{abstract}
Malaria is a major and growing threat to economic development and public health in developing countries. There are about 2.7 million malaria deaths annually. Plasmodium knowlesi, the fifth human malaria parasite, is an attractive model for malaria research and is also phylogenetically close to human malaria parasite $P$. vivax. It shares many vaccine candidate molecules with $P$. vivax. This study was carried out to determine immunological profiles mounted by Olive baboons during experimental infection with $P$. knowlesi $\mathrm{H}$ strain. Six Olive baboons (Papio Anubis) were infected with $1 \times 10^{6}$ P. knowlesi blood stage parasites. The infected baboons developed
\end{abstract}


either severe (acute) or mild (chronic) infection. High IgM titres were observed during primary infection in the baboons. Anti-IgG antibodies were raised after two weeks of chronic infection. Circulating IFN $\gamma$ from serum rose fifty fold in acutely infected animals during the first two weeks post infection compared to a tenfold increase in chronically infected baboons. Recall proliferative responses were two fold higher in chronically infected animals by day 14 post infection. The highest stimulation index $(10.06 \pm 2.74)$ was observed in chronic animals at day 42 post infection. These results demonstrated that baboons infected with $P$. knowlesi mount an immune response that is characterized by antibody and cytokine responses involving $\operatorname{IgG}, \operatorname{IgM}$ and IFN $\gamma$. These studies are important in validation of the $P$. knowlesi- baboon model for malaria drug and vaccine development.

Keywords: Plasmodium knowlesi, Olive Baboons, Immune responses

\section{Introduction}

Evaluation of new drugs and vaccines for use in humans requires testing in animal models that develop a disease comparable to that in humans. Similarities in biological mechanisms between human and non-human primates underlie the value of non-human primates as the final test systems preceding clinical trials in humans. The baboon is an attractive experimental model because it is well characterized and is also frequently used in biomedical research (King et al., 1988). Baboons are fully susceptible to Plasmodium knowlesi infections (Coatney et al., 1971; Ozwara et al., 2003). In fact, the disease profile observed in baboons is similar to that in rhesus monkeys following infection with the same parasite strain (Ozwara et al., 2003). Papio anubis infected with $P$. knowlesi shows various clinical characteristics that are also seen in human malaria, including cerebral involvement (Ozwara et al., 2003; Aikawa et al., 1990; Aikawa., 1988).

Plasmodium knowlesi infects animals on multiple occasions before immunity develops and interactions can be evaluated in immunological and metabolic environment similar to humans. The parasite was instrumental in the discovery and characterization of malaria blood stage vaccine candidate AMA-1 (Kocken et al., 1999). In fact $P$. knowlesi has been used to identify, develop, and evaluate vaccine and drug candidates (Wengelnik et al., 2002; Kocken et al., 1999; Deans et al., 1984). Protocols for genetic modification of $P$. knowlesi have been developed (Kocken et al., 2002). These are powerful tools for drug and vaccine development because they enable the functions of target drug and vaccine candidates to be determined using P. knowlesi genes (Ozwara et al., 2003).

As the largest monkey available for malaria research, the baboon provides a primate host for use in in vivo experiments for understanding malaria parasite biology and developing control measures against malaria. Host parasite interactions of both the wild type and the transfected $P$. knowlesi parasites can be readily studied (Ozwara, et al., 2003; Munyao et al., 2008).

At the moment the immune responses mounted by $P$. anubis against $P$. knowlesi have not been described. The analysis of the immune responses of the baboons to $P$. knowlesi infections is important in the development and validation of the baboon model for malaria research. The described study looks at some of the immune response profiles following experimental $P$. knowlesi $\mathrm{H}$ strain infections in baboons.

\section{Methods}

\subsection{Parasites}

P. knowlesi $\mathrm{H}$ strain (Ozwara et al., 2003) blood stage parasites were used for inducing baboon infection. These parasites were retrieved from liquid nitrogen and quickly thawed at $37^{\circ} \mathrm{C}$ in a water bath, washed twice in $3.5 \%$ $\mathrm{NaCl}$ and twice in RPMI 1640 (sigma, UK) by spinning at 1200 revolutions per minute (RPM) for 10 minutes. The pellet was then transferred into a starting culture medium consisting $2.5 \%$ packed cell volume (PCV), $20 \%$ baboon serum and the rest RPMI 1640 having $20 \mu \mathrm{g} / \mathrm{ml}$ of gentamycin (Sigma, UK). The parasites were cultured overnight before being inoculated into baboons.

\subsection{Animals}

Six adult baboons ( $P$. anubis, weight range of $12-23 \mathrm{~kg}$ ) were used. Prior to the experiments, all animals were screened and determined to be free of infection with Plasmodium by giemsa-stained thick blood film. All the animals were fed on a standard diet for non -human primates and water was provided ad libitum (Olobo and Reid, 1990). The institutional animal care and use committee, and the institutions scientific and ethical review committee (ISERC) of the Institute of Primate Research approved the experiments. The baboons were selected and housed individually in single cages in a bio-containment facility to avoid possible transmission of $P$. knowlesi between experimental baboons. 


\subsection{Animal Infection, observation and sampling}

All the six animals were sedated using ketamine hydrochloride (vetelar, parnke Davies co., ponypool Germany) at $10 \mathrm{mg} / \mathrm{kg}$ body weight. They were then inoculated intravenously with $1 \times 10^{6}$ P. knowlesi blood stage parasites in $1 \mathrm{ml}$ of RPM1 1640 medium (Invitrogen, UK). Prior to inoculation, $13 \mathrm{mls}$ of blood was obtained from each animal, $10 \mathrm{ml}$ was obtained in alsevers solution and used for peripheral blood mononuclear cells preparation and the rest was processed for serum. This was used to get pre-infection values for IFN $\gamma$ levels, antibody titres and recall proliferative responses. These formed the baseline values over the other subsequent values obtained from each animal in the other sampling points. Animals were then bled on day 7 and day 14 post infection. Acute animals (animals that had parasitaemia systematically rise to over $6 \%$, reaching as high as $9.1 \%$ in some baboons at the time of treatment) were treated when parasitaemia reached above $6 \%$; and chronic animals (animals that had peak parasitaemia levels ranging from $1.5 \%-5 \%$, which thereafter decreased to less than $2 \%$ parasitaemia on average) were treated on day 11 post infection irrespective of parasitaemia. Each of the groups had three animals. Treatment was done using pyrimethamine (5-[4-chrophenyl]-6-ethyl-2, 4-pyrimidinediamine; sigma aldrichemie, UK) at $1 \mathrm{mg} / \mathrm{kg}$ body weight once per day for four days. Finger pricks were taken from each animal on a daily basis from day three up to the day the animal was cured and used to prepare blood film which was used to determine the parasitaemia levels. Parasitaemia was determined as described in Ozwara et al., 2003.

Chronic animals were then challenged two weeks after treatment and confirmation of cure. Challenge was done with a similar number of parasites as described above. Finger pricks and bioassay similar to those done for the initial infection were repeated. The general status of the animals was monitored by regularly determining weight, appetite and general behavior. Weight of the animals was measured on a sedated animal at the bleeding time points. Appetite was evaluated by closely monitoring the feeding habits of the animals.

\subsection{Antibody detection}

Polystylene Micro ELISA plates (Dynatech laboratories, Sussex, UK) were coated overnight with $100 \mu 1$ of crude and solubilized whole parasite homogenised antigen at a concentration of $1 \times 10^{6}$ parasites $/ \mathrm{ml}$ diluted in bicarbonate buffer, $\mathrm{pH}$ 9.6. Excess coating buffer was flicked off and non-specific binding sites were blocked with $3 \%$ bovine serum albumin (BSA Sigma, UK) in PBS for $1 \mathrm{hr}$ at $37^{\circ} \mathrm{C}$. Unbound BSA was washed off six times with $0.05 \%$ Tween 20 (Sigma, UK) in PBS. Fifty microlitres of undiluted serum samples were then dispensed into the wells and incubated for 2 hour at $37^{\circ} \mathrm{C}$. Control wells received $50 \mu \mathrm{l}$ of the blocking buffer. Unbound serum was washed off six times as above and $50 \mu$ of alkaline phosphate conjugated rabbit anti monkey $1 \mathrm{gG}$ (Cappel, organon Teknika, Or,USA) diluted 1/2000 was added and followed by incubation for 1 hour at $37^{\circ} \mathrm{C}$. Unbound conjugated rabbit anti monkey $\operatorname{IgG}$ was washed off as above before adding $50 \mu \mathrm{l}$ of p-nitrophenyl phosphate substrate (pNPP, Sigma, UK, final concentration $1 \mathrm{mg} / \mathrm{ml}$ ) in $10 \%$ diethanolamine buffer. The plates were incubated at $37^{\circ} \mathrm{C}$ for 30 minutes in the dark. Optical density (O.D) was read at $405 \mathrm{~nm}$ in a microplate reader (Dynatech laboratories, UK). In assaying for IgM antibodies in serum, $100 \mu \mathrm{l}$ of Horse radish peroxidase conjugated rabbit anti monkey IgM (cappel Organon Teknika, OR, USA) diluted 1/2000 was added and incubated for one hour at $37^{\circ} \mathrm{C}$. One hundred microlitres Orthophenyldiamine (OPD Sigma, UK, final concentration of $0.4 \mu \mathrm{g} / \mathrm{ml}$ ) in phosphate citrate buffer was used as the substrate. Optical density (O.D) was read at $450 \mathrm{~nm}$ after $30 \mathrm{~min}$ of incubation.

\subsection{Interferon gamma detection}

Purified peripheral blood mononuclear cells (PBMC) were adjusted to $4 \times 10^{6} \mathrm{ml}$ in complete medium -RPMI 1640 medium supplemented with $10 \%$ heat inactivated FBS, 2-mercaptoethanol $\left(5 \times 10^{-5} \mathrm{M}\right)$, L-glutamine $(5 \mathrm{mM} / \mathrm{ml})$, and gentamycin $(100 \mu \mathrm{g} / \mathrm{ml})$ and stimulated with either Con A or crude $P$. knowlesi whole parasite antigen. Culture supernatants were pooled from triplicate wells after the stimulation of PBMC. The concentration of IFN $\gamma$ in the supernatants produced after the 72 hours of stimulation, and serum samples was determined in a sandwich ELISA as follows:

Polystyrene micro-Elisa plates (Dynatech laboratories, Sussex UK) were coated overnight with $50 \mu$ l of monoclonal antibody which was rabbit anti-human recombinant IFN $\gamma(2 \mu \mathrm{g} / \mathrm{ml}$; Genzyme Corporation MA, USA). The wells were washed 6 times with washing buffer (1\%BSA and $0.05 \%$ Tween 20 in PBS). Non specific binding sites were blocked with $200 \mu \mathrm{l}$ of $3 \%$ BSA in PBS for $1 \mathrm{hr}$ at $37^{\circ} \mathrm{C}$. Excess blocking buffer was flicked off, and $50 \mu \mathrm{l}$ of test samples added. Wells where blocking buffer alone was added (blank wells) served as negative controls. These were incubated for $2 \mathrm{hrs}$ at $37^{\circ} \mathrm{C}$ and then washed as described above. $50 \mu \mathrm{l}$ biotinylated anti-human IFN $\gamma$ (Mab-7B6-1-Biotin: MABTECH, Sweden) was then added at a dilution of 1:200 per well and incubated for $1 \mathrm{hr}$ at $37^{\circ} \mathrm{C}$. Washing was done as before and $50 \mu \mathrm{l}$ of streptoavidin (ALP-PQ MABTECH, Sweden) was added at a dilution of 1:10,000 to each well, and incubated for $1 \mathrm{hr}$ at $37^{\circ} \mathrm{C}$. Fifty microlitres of p-NPP in $10 \%$ 
diethanolmine buffer was added to each well and plates incubated at $37^{\circ} \mathrm{C}$ for 30 minutes in the dark. The plates were read at an Optical density (O.D) of $405 \mathrm{~nm}$ in a microplate reader. Optical density readings for the blank wells were subtracted from O.D readings of the sample wells to get the final O.D reading.

\subsection{Recall proliferation assay}

Peripheral blood was obtained from the femoral vein and diluted in an equal volume of sterile Alsevers solution. Peripheral blood mononuclear cells (PBMC) were isolated over Ficoll-hypaque by centrifugation at 3000 RPM for 30 minutes. A ratio of $4 \mathrm{ml}$ of diluted blood to $3 \mathrm{ml}$ of Ficoll-hypaque was used. Cells at the interface were aspirated and washed in Alsevers solution and complete RPM1 1640 medium. Cell viability was determined by trypan blue stain.

Purified PBMC were adjusted to $4 \times 10^{6} / \mathrm{ml}$ in complete RPM1 1640 medium. Cell suspension $(50 \mu \mathrm{l})$ was distributed to each well of the 96 well flat bottomed micro titre plates (Nunc, Roskilde, Denmark). One hundred microlitres of crude P. knowlesi whole parasite antigen ( $1 \times 10^{6}$ parasites) or Concanavalian A (Con A; sigma UK) at a concentration of $10 \mu \mathrm{g} / \mathrm{ml}$ were added to the wells. Control wells received $100 \mu \mathrm{l}$ of complete RPM1 1640 . Cultures were set up in triplicates.

The cultures were then incubated at $37^{\circ} \mathrm{C}$ in humidified atmosphere containing $5 \% \mathrm{CO}_{2}$ for 72 hours. The cells were pulsed with $0.5 \mu \mathrm{Ci}$ of ${ }^{3} \mathrm{H}$-thymidine $(5 \mathrm{~m} \mathrm{Ci} / \mathrm{mg}$ : Amersham, UK) in $20 \mu \mathrm{RPM} 11640$ medium and a further incubation was carried out for 18-24hrs. Cells were harvested onto fibre glass filters by using Titertek cell harvesters (Flow laboratories, UK). The filters were air dried and immersed in scintillation fluid (PPO, POPOP, Triton $\mathrm{x}-100$, Germany). Incorporation of ${ }^{3} \mathrm{H}$-thymidine into DNA was determined by liquid scintillation spectrophotometry. Results were expressed as stimulation index (SI) that was obtained by dividing the proliferation of test cultures by proliferation of control cultures.

\section{Results}

\subsection{Primary infection parasitaemia}

All the baboons inoculated with $P$. knowlesi $\mathrm{H}$ strain blood stages developed patent parasitaemia with all the blood stages being manifested. Those that had acute parasitaemia, developed patent parasitaemia by day four post inoculation while chronic animals had patent parasitaemia by day five inoculation (Table 1). Acute animals had parasitaemia that systematically increased to over $6 \%$, reaching as high as $9.1 \%$ in some baboons at the time of treatment. Chronic animals had peak parasitaemia levels ranging from 1.5\%-5\%, which thereafter decreased to less than $2 \%$ parasitaemia on average.

\subsection{Secondary infection parasitaemia}

Animals with chronic parasitaemia were cured and re-infected as described earlier. The animals developed patent parasitaemia by day six post infection. Peak parasitaemia levels were on average, lower than during the primary infection (Table 2). Thereafter parasitaemia levels decreased to sub patent levels by day 14 post re-infection. Animals that had developed acute parasitaemia during the primary infection were withdrawn from the experiment due to technical reasons unrelated to the experiment

\subsection{Clinical symptoms}

The clinical symptoms observed were as seen by Ozwara and others (Ozwara et al., 2003).

\subsection{Antibody responses}

All baboons produced anti $P$. knowlesi $\operatorname{IgG}$ and IgM antibodies upon infection with $P$. knowlesi blood stage parasites (Figure 1 and Figure 2). During the first two weeks of infection, IgM responses were higher in acute animals (Mean O.D \pm SD: $0.238 \pm 0.135$ ) than in the chronically infected animals (Mean O.D $\pm S D: 0.173 \pm 0.057$ ). Anti $P$. knowlesi $1 \mathrm{gG}$ responses increased slowly upon infection. The increase in $\mathrm{IgG}$ responses however was higher in chronic animals (Mean O.D \pm SD: $0.113 \pm 0.04$ ) than in acute animals (Mead O.D \pm SD: $0.097 \pm 0.014$ ). Immunoglobulin $\mathrm{G}$ levels had increased by $18 \%$ in acute animals at day 14 post infection, whereas in chronic animals the increase at day 14 post infection was $48 \%$, and $72 \%$.. Immunoglobulin $\mathrm{M}$ levels in both the acutely and chronically infected baboons remained higher than IgG levels in all the sampling points, apart from day 42 where the IgG levels were higher in the chronic animals (Figure 2).

\subsection{Cytokine responses}

All the animals produced IFN $\gamma$ by day 7 post infection (Figure 3). Acute animals showed a steady increase in serum IFN $\gamma$ (Figure 3). Although the IFN $\gamma$ produced by animals with chronic infection also rose in the course of the primary infection reaching a peak at day 14 (Figure 3), the levels in acutely infected animals was much 
higher at the two sampling points (Figure 3). Upon treatment, the IFN $\gamma$ levels in chronically infected animals subsided and then rose again one week later, only to decrease again by day 42 post infection (Figure 3 ). Interferon gamma detected in blast supernatants increased in both acute and chronically infected animals upon infection (Figure 4). The levels in acutely infected animals reached peak by day 14 post infection (Figure 4). In chronically infected animals, IFN $\gamma$ in the supernatant increased gradually to reach a peak at day 42 post primary infection (Figure 4). Levels of IFN $\gamma$ produced by cells from acutely infected animals remained higher than in those from animals with chronic infection (Figure 4).

\subsection{Cellular immune responses}

PBMC from the baboons showed mitogen and antigen specific proliferation, with the highest stimulation index observed from chronically infected animals by day 42 post primary infection $10.06+2.74$ (mean \pm S.D). Antigen proliferation observed in PBMC from acutely infected animals was lower compared to chronically infected animals at day 14 post inoculation (Figure 5). Upon re-infection, antigen specific proliferation responses increased in chronically infected animals by day 14 post re-infection (Figure 5).

\section{Discussion}

In this study immune responses (antibody, cytokine and recall proliferation responses) mounted by experimental $P$. knowlesi infection in olive baboons (Papio anubis) are presented for the first time. Our report confirms that $P$. anubis is fully susceptible to experimental $P$. knowlesi $\mathrm{H}$ strain infection since all infected animals developed patent parasitaemia. Infected baboons developed either acute or chronic infection. Acutely infected baboons mounted an immune response that failed to control parasitaemia. Previous work showed that $P$. knowlesi infected baboons develop either acute or chronic infection (Ozwara et al., 2003). Results in the current study are in support of the findings in the above mentioned previous study. The precise mechanism that predisposes a dual outcome in the infection profile warrants further investigations.

In baboons with severe $P$. knowlesi infection, patent parasitaemia developed earlier than in the chronically infected animals. The disease profiles reported here are similar to rhesus monkeys infected with $P$. knowlesi $\mathrm{H}$ strain (Ozwara et al., 2003; Ibiwoye et al., 1993; Rosen et al., 1998). However, our experiments are the first to show that $P$. knowlesi infected baboons can be infected, treated and re-infected. This suggests that baboons infected with $P$. knowlesi do not develop sterile infection following challenge exposure. Although it has been established that immune responses against malaria does not result in sterile infection (Chen et al., 2000), we have shown that chronically infected $P$. anubis are able to control parasitaemia to sub patent levels upon re-infection. The profile of secondary infection of acutely infected baboons remains unknown. Secondary infection data from these animals indicates that protection is associated with pre-exposure as described in Voller and Rossan, 1969 and Richie and Saul, 2002.

During primary infection, antibody (IgG and IgM) levels increased in both infection groups. Immunoglobin $\mathrm{M}$ titres increased steadily and remained higher than the IgG levels. Levels of IgM were raised more rapidly during the primary infection. In addition acutely infected animal showed higher IgM levels. Plasmodium knowlesi infections in monkeys show antigenic variations (Brown K and Brown I, 1995). This antigenic variation is possibly responsible for this persistently raised IgM levels seen in the P. knowlesi infected baboons. It is interesting to note that acutely infected animal produce higher IgM titres while chronically infected baboons show elevated IgG production. This pattern suggests that elevated production of IgM on its own is not sufficient to control parasitaemia and prevent severe infection. Previous studies have shown that IgM responses are mounted faster than IgG, especially in primary infections and IgM is seen to boost the initial response (Roit, 1988). This is similar to our findings. However, we can report here that control of parasitaemia is associated with elevated IgG response, as shown in chronically infected animals. The rise in IgG levels was modest during primary infection but elevated following challenge exposure of chronically infected animals, showing evidence of immunological memory.

Acutely infected animals developed higher parasitaemia levels as compared to the chronic animals during the primary infection. The fact that chronically infected animals were able to control the parasitaemia during the primary infection and reduce parasitaemia further to below detectable levels upon re-infection supports the role of acquired immune responses in protection against $P$. knowlesi infected baboons. It has been demonstrated in human malaria blood stage infection that antibodies are involved in protection (Miller et al., 1998; Good et al., 1998). Furthermore, T cell proliferation in response to blood stage antigens is also associated with protection (Miller et al., 1998; Good et al., 1998). In addition there is growing evidence for the protective role of IgG in $P$. falciparum (Christophe et al., 2000). Passive transfer of IgG has provided protection against $P$. falciparum blood stage in South American monkeys (Fandeur et al., 1984; Groux and Gysin, 1990), and in humans 
(Bouharoun-Tayoun et al., 1990). Furthermore, human antibodies efficiently inhibit in vitro P. falciparum merozoite proliferation (Bouharoun-Tayoun et al., 1990), and mediate opsonisation of infected erythrocytes (Groux and Gysin, 1990). Our study indicates a role for IgG in protection against severe disease in baboons. However, further investigations are warranted to determine the role of increased IFN $\gamma$ production in acute infection as shown in this study.

During the primary infection, proliferative cellular responses were higher in chronically infected baboons, especially after two weeks post-infection. This clearly demonstrates better immunological memory elicited in chronically infected baboons. Because antigen induced proliferative responses are pre-dominantly mediated by $\mathrm{T}$ cells, our results suggest that $\mathrm{T}$ cell memory develops in $P$. knowlesi infected baboons. This can be interpreted further to mean that $\mathrm{T}$ cell responses are required in order to control parasitaemia in these animals. Similar suggestions have been made for studies in humans and chimpanzees (Pombo et al., 2002; Taylor et al., 1985).

The role of cytokines in the regulation of immune responses against Plasmodium infections and pathogenesis of malaria has been extensively studied in rodent malaria models but there are few studies in primate malaria (Chunfu yang et al., 1999). In one study in which human volunteers were infected experimentally with Plasmodium falciparum, there was increased production of IFN $\gamma$ (Harpaz et al., 1992). In a second study, $P$. Coatneyi infected Rhesus monkeys demonstrated an increased IFN $\gamma$ production occurring in the early stage of infection, and is thought to have been associated with the pathologic changes that led to death of the infected animals (Chunfu yang et al., 1999). This observation is supported by Riley (1990) who suggested that low amounts of IFN $\gamma$ may be associated with resolution of infection. Therefore, excessive production of proinflammatory cytokines by human host has an adverse effect on disease progression (Chen et al., 2000). High circulating levels of IFN $\gamma$ and TNF $\alpha$ are more often found in patients with severe malaria than in uncomplicated cases (Kwiatkowski et al., 1990). These studies, combined with ours, demonstrate a negative role for high IFN $\gamma$ production during primary malaria infection. Resistance or susceptibility to malaria may depend on induction of appropriate levels of IFN $\gamma$ and possibly other pro-inflammatory cytokines like TNF $\alpha$ as suggested by others (Riley, 1990). Factors that mediate high IFN $\gamma$ production early in baboons, resulting in severe disease warrants further investigation. Similar mechanisms are likely operating in humans infected with P. falciparum. We can conclude that this phenomenon is linked to dual disease outcomes observed in baboons infected with $P$. knowlesi. Described results are important in validation of the new baboon model for drug and vaccine development against malaria.

\section{Conclusion}

In this study, baboons experimentally infected with $P$. knowlesi and showing acute infection profile were shown to produce higher IFN $\gamma$ in the first two weeks of infection compared to chronically infected animals. This has indicated that high IFN $\gamma$ production early in infection is associated with severe disease outcome. Although early infection in both acute and chronically infected baboons was associated with high IgM responses, antibody on its own was unable to prevent development of severe infection. Mechanisms resulting in moderated production of IFN $\gamma$ at the start of malaria infection in baboons partly hold the key in understating control of severe infection. These studies are important in validation of the $P$. knowlesi- baboon model for malaria drug and vaccine research

\section{Authors Contributions}

T. Nyawira planned the study, undertook laboratory experiments and wrote the draft manuscript. M. Gicheru and Z. Ng'ang'a participated in supervising experiments and reviewing the manuscript. H. Ozwara developed the idea, obtained funding for the study and supervised planning, experiments and reviewed the manuscript.

\section{Acknowledgements}

We thank head of department, veterinary staff and technicians of the animal sciences department, Institute of Primate Research for assisting with baboon infection and sampling.

\section{References}

Aikawa, M. (1988). Human cerebral malaria. American Journal of Tropical Medicine and Hygiene, 39, 3-10.

Aikawa, M., Iseki, M., Barnwel, J. W., Taylor, D., Oo, M. M., \& Howard, R. J. (1990). The pathology of human cerebral malaria. American Journal of Tropical Medicine and Hygiene, 43, 30-37.

Bouharoun-Tayoun, H., Attanath, P., Sabchareon, A., Chong, S. T., \& Druihe, P. (1990). Antibodies that protect humans against Plasmodium falciparum blood stages do not on their own inhibit parasite growth and invasion in vitro but act in co-operation with monocytes. Journal of Experimental Medicine, 172, 1633-1641. http://dx.doi.org/10.1084/jem.172.6.1633 
Brown, K., \& Brown, I. N. (1965). Immunity to malaria: antigenic variation in chronic infections of Plasmodium knowlesi. Nature, 208, 1286-1288. http://dx.doi.org/10.1038/2081286a0

Chen, Q., Schlichtherle, M., \& Wahlgren, M. (2000). Molecular aspects of severe malaria. Clinical Microbiology Reviews, 13(3), 439-450. http://dx.doi.org/10.1128/CMR.13.3.439-450.2000

Christophe, A., Traore, Y., Tall, F., Nacro, B., Therese, T. L., Fumoux, F., \& Rihet, P. (2000). High immunoglobulin G2 (IgG2) and low IgG4 levels are associated with human resistance to Plasmodium falciparium malaria. Infection and Immunity, 68, 1252-1258. http://dx.doi.org/10.1128/IAI.68.3.1252-1258.2000

Chunfu, Y., Lihua, X., Jon, E., Joann, S., Altaf, A., \& Collins, W. (1999). Cytokine production in rhesus monkeys infected with Plasmodium coatneyi. American Journal of Tropical Medicine and Hygiene, 61, 226-229.

Coatney, G. R., Collins, W. E., Warren, M., \& Contracos, P. G. (1971). The primate malarias. Bethesda, Md: US Govt Print Off Washington DC x-366.

Deans, J. A., Thomas, A. W., Alderson, T., \& Cohens, S. (1984). Biosynthesis of a putative protective Plasmodium knowlesi merozoite antigen. Mol Biochem Parasitol, 11,189-204. http://dx.doi.org/10.1016/0166-6851(84)90065-3

Fandeur, T., Dubois, P., Gysin, J., Dedet, J., \& Da silva, L. P. (1984). In vitro and in vivo studies of protective and inhibitory antibodies against Plasmodium falciparum in saimiri monkey. Journal of Immunology, 132, 432-437.

Good, M. F., Kaslow, D. C., \& Miller, L. H. (1998). Pathways and strategies for developing a malaria blood stage vaccine. Annual Review of Immunology, 16, 57-87. http://dx.doi.org/10.1146/annurev.immunol.16.1.57

Groux, H. \& Gysin, J. (1990). Opsonisation as an effector mechanism in human protection against asexual blood stages of Plasmodium falciparum: functional role of IgG subclasses: Immunologic Research, 141, 529-542. http://dx.doi.org/10.1016/0923-2494(90)90021-P

Harpaz, R., EdeIman, R., Wasserman, S. S., Levine, M. M., Davis, J. R., \& Sztein, M. B.(1992). Serum cytokine profiles in experimental human malaria - relationship to protection and disease course after challenge. Journal of Clinical Investigation, 90, 515-523. http://dx.doi.org/10.1172/JCI115889

Ibiwoye, M., Howard, C., Sibbons, P., Hassan, M., \& Van velzen, D. (1993). Cerebral malaria in rhesus monkeys (Macaca Mulatta): observations on host pathology. Journal of Camparative Pathology, 108, 303-310. http://dx.doi.org/10.1016/S0021-9975(08)80293-9

King, F. A., Yarbrough, C. J., Anderson, D. C., Gordon, T. P., \& Gould, K. G. (1998). Primates. Science, 240, 1475-1482. http://dx.doi.org/10.1126/science.3287624

Kocken, C. H., Dubbled, M. A., van der Wel, A., Pronk, J. T., Waters, A. P., Langermans, J. A., \& Thomas A. W. (1999). High level expression of P. vivax apical membrane antigen (AMA - I) in Pichia pastoris; strong immunogenicity in M. mulatta immunized with $P$. vivax AMA-I and adjuvant SBAS2. Infection and Immunity, 67, 43-49.

Kocken, C. H., Ozwara, H., van der Wel, A., Beetsma, A. L., Mwenda, J. M., \& Thomas, A. W. (2002). Plasmodium knowlesi provides in vitro and in vivo transfection system that enables double cross over gene knock out studies. Infection and Immuity, 70, 655-660. http://dx.doi.org/10.1128/IAI.70.2.655-660.2002

Kwiatkowski, D., Hill, A. V., Sambou, I., et al. (1990). TNF concentration in fatal cerebral, non fatal cerebral, and uncomplicated Plasmodium falciparun malaria. Lancet, 336, 1201-1204. http://dx.doi.org/10.1016/0140-6736(90)92827-5

Miller, L. H., Good, M. F., \& Kaslow, D. C. (1998). Vaccines against the blood stages of falciparium malaria. Advances in Experimental Medicine and Biology, 452, 193-205. http://dx.doi.org/10.1007/978-1-4615-5355-7_22

Munyao, J. M., Gicheru, M. M., Ng'aNg'a, Z., Kagasi, E., \& Ozwara, H. S. (2008). Transfection of Plasmodium knowlesi in baboons (Papio anubis) provides a new system for analysis of parasites expressed transgenes of host parasite interface. International Journal of Integrative Biology, 2,100-108.

Olobo, J. \& Reid, G. (1990). Mitogenic responses of peripheral mononuclear cells of vervet monkeys (Cercopithecus aethiops): apparent role of adherent cells. American Journal of Primatology, 20:31-36. http://dx.doi.org/10.1002/ajp.1350200105 
Ozwara, H., Langermans, J. A., Maamun, J., Farah, Yole, D. S., Mwenda, J. M., Weiler, H., \& Thomas, A. W. (2003). Experimental infection of the olive baboon (Papio anubis) with Plasmodium knowlesi: severe disease accompanied by cerebral involvement. American Journal of Tropical Medicine and Hygiene, 69, 188-194.

Pombo, D., Gregor, L., Chakrit, C., et al. (2002). Immunity to malaria after administration of ultra low doses of red cells infected with Plasmodium falciparum. Lance, 360, 610-617 http://dx.doi.org/10.1016/S0140-6736(02)09784-2

Richie, T. L., Allan, S. (2002). Progress and challenges for malaria vaccines. Nature, $415,694$. http://dx.doi.org/10.1038/415694a

Riley, M. E. (2002). Is T cell priming required for initiation of pathology in malaria infections? Immunology Today, 20, 228-233. http://dx.doi.org/10.1016/S0167-5699(99)01456-5

Roitt, M. (1988). Essential immunology. Blacwell scientific publications, 85-100.

Rosen, S., Hano, J., \& Barry, K. (1968). Malaria neuropathy in rhesus monkeys. Archives of Pathology and laboratory medicine, 85, 36-44.

Taylor, D., Wells, R., Vernes, A., Yvonne, J., Vogel, S., \& Diggs, C. (1985). Parasitologic and immunologic studies of experimental Plasmodium falciparum infection in non splenectomised Chimpanzees (Pan troglodytes). American Journal of Tropical Medicine and Hygiene, 34, 36-44.

Voller, A. \& Rossan, R. (1969). Immunological studies of simian malaria: immunity to challenge and antigenic variation in P. knowlesi. Transactions of the Royal Society of Tropical Medicine and Hygiene, 63, 507-523. http://dx.doi.org/10.1016/0035-9203(69)90040-6

Wengelnik, K., Vidal, V., Ancelin, M. I., et al. (2002). A class of potent antimalarials and their specific accumulation in infected erythrocytes. Science, 295, 1311-1314. http://dx.doi.org/10.1126/science.1067236

Table 1. Disease status of baboons infected with $P$. knowlesi H Strain during primary infection

\begin{tabular}{|c|c|c|c|}
\hline \multirow{2}{*}{$\begin{array}{l}\text { Baboon } \\
\text { number }\end{array}$} & Infection & profile & \multirow{2}{*}{$\begin{array}{l}\text { Day for } \\
\text { patent } \\
\text { parasitaemia }\end{array}$} \\
\hline & Profile & $\begin{array}{l}\text { Day post } \\
\text { inoculation }\end{array}$ & \\
\hline Pan 2730 & Acute & 9 & 3 \\
\hline $\operatorname{Pan} 2574$ & Acute & 9 & 3 \\
\hline Pan 2677 & Acute & 10 & 4 \\
\hline $\operatorname{Pan} 2601$ & Chronic & 10 & 5 \\
\hline Pan 2669 & Chronic & 10 & 5 \\
\hline Pan 2698 & Chronic & 11 & 5 \\
\hline
\end{tabular}


Table 2. Comparison of parasitaemia in chronic animals during primary and secondary infections with $P$. knowlesi

\begin{tabular}{|c|c|c|c|}
\hline $\begin{array}{l}\text { Baboon } \\
\text { number }\end{array}$ & Parameter & $\begin{array}{l}\text { Primary } \\
\text { infection }\end{array}$ & $\begin{array}{l}\text { Secondary } \\
\text { infection }\end{array}$ \\
\hline \multirow[t]{3}{*}{ Pan 2669} & Day of patent parasitaemia & 5 & 6 \\
\hline & Peak parasitaemia & $5 \%$ & $2.39 \%$ \\
\hline & Treatment parasitaemia $^{*}$ & $1.3 \%$ & $<<$ \\
\hline \multirow[t]{3}{*}{ Pan 2601} & Day of patent parasitaemia & 5 & 6 \\
\hline & Peak parasitaemia & $2.6 \%$ & $1.5 \%$ \\
\hline & Treatment parasitaemia $^{*}$ & $1.94 \%$ & $<<$ \\
\hline \multirow[t]{3}{*}{ Pan 2698} & Day of patent parasitaemia & 5 & 6 \\
\hline & Peak parasitaemia & $1.5 \%$ & $2.62 \%$ \\
\hline & Treatment parasitaemia $^{*}$ & $0.1 \%$ & $<<$ \\
\hline
\end{tabular}

$<<$ Parasitaemia below detectable levels.

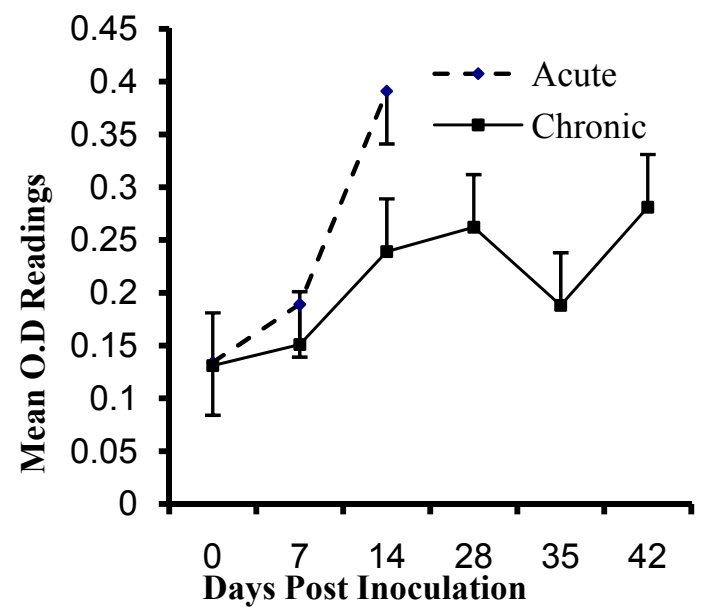

Figure 1. Mean IgM responses of baboons infected with P. knowlesi

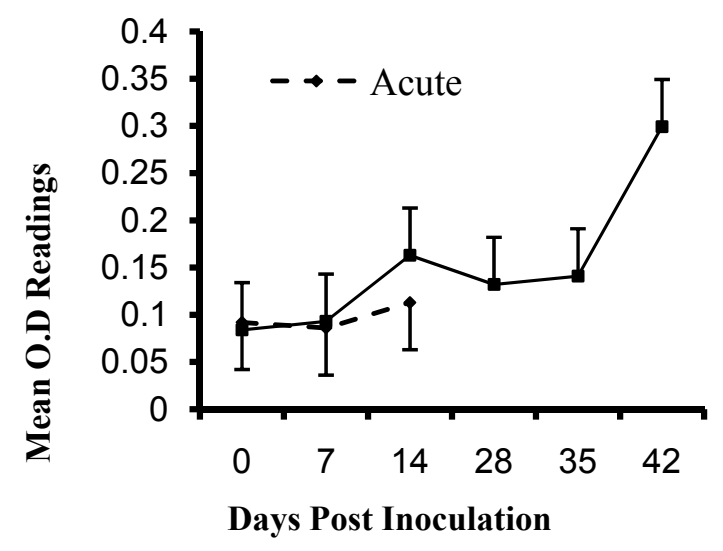

Figure 2. Mean IgG responses of baboons infected with $P$. knowlesi 


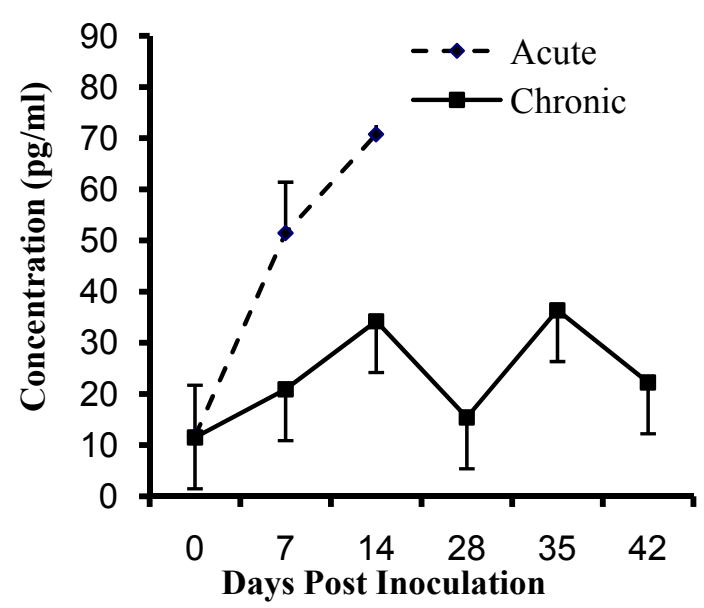

Figure 3. Concentration of IFN- $\gamma$ in serum from baboons infected with $P$. knowlesi

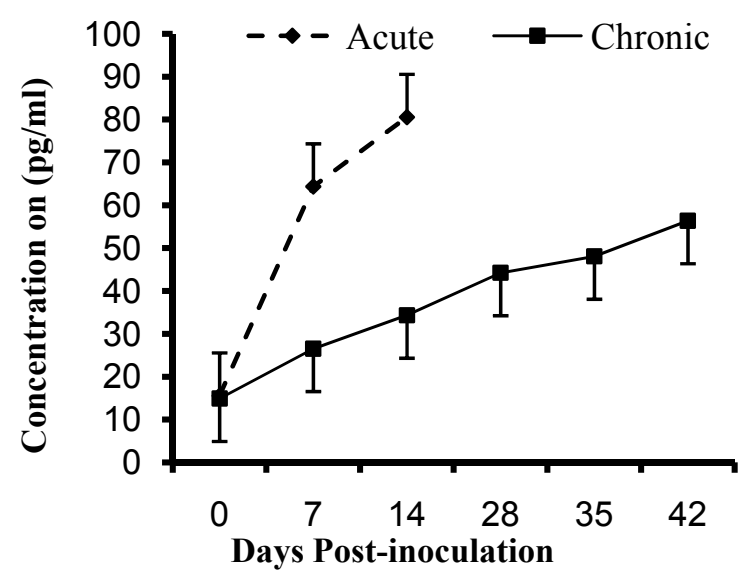

Figure 4. Interferon gamma levels in supernatants from PBMC stimulated with $P$. knowlesi crude whole parasite antigen

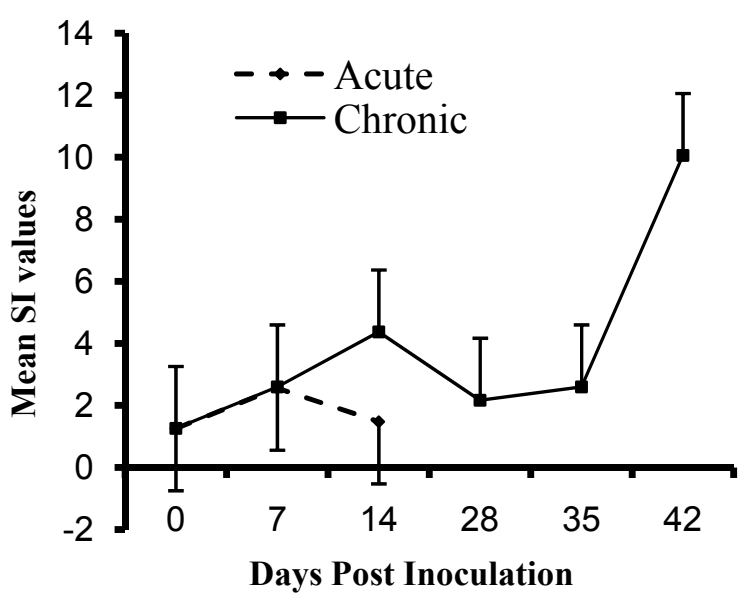

Figure 5. Antigen specific recall proliferative responses of PBMC from P. knowlesi infected baboons 